PROCEEDINGS OF THE

AMERICAN MATHEMATICAL SOCIETY

Volume 133, Number 2, Pages 441-448

S 0002-9939(04)07563-X

Article electronically published on August 25, 2004

\title{
TIETZE EXTENSION THEOREM FOR HILBERT $C^{*}$-MODULES
}

\author{
DAMIR BAKIĆ
}

(Communicated by David R. Larson)

\begin{abstract}
We prove the following generalization of the noncommutative Tietze extension theorem: if $V$ is a countably generated Hilbert $C^{*}$-module over a $\sigma$-unital $C^{*}$-algebra, then the canonical extension $\bar{\Phi}$ of a surjective morphism $\Phi: V \rightarrow W$ of Hilbert $C^{*}$-modules to extended (multiplier) modules, $\bar{\Phi}: V_{d} \rightarrow W_{d}$, is also surjective.
\end{abstract}

\section{INTRODUCTION AND STATEMENT OF THE RESULTS}

Given a surjective morphism of $C^{*}$-algebras $\varphi: \mathcal{A} \rightarrow \mathcal{B}$, there exists a morphism $\bar{\varphi}: M(\mathcal{A}) \rightarrow M(\mathcal{B})$, an extension of $\varphi$ to the corresponding multiplier algebras. There is a purely algebraic construction of $\bar{\varphi}$ by means of double centralizers (cf. [7], Proposition 2.2.16). Another way to display $\bar{\varphi}$ is to regard $\mathcal{A}$ and $\mathcal{B}$ as Hilbert $C^{*}$ modules then $M(\mathcal{A})$ and $M(\mathcal{B})$ are interpreted as the $C^{*}$-algebras of adjointable operators on $\mathcal{A}$ and $\mathcal{B}$, respectively ([5]; see also the proof of Proposition 3 below). It turns out that $\bar{\varphi}$ is the extension of $\varphi$ by strict continuity, hence $\bar{\varphi}$ is the unique (canonical) extension of $\varphi$ to multiplier algebras.

The noncommutative Tietze theorem, first proved by G. Pedersen, asserts that $\bar{\varphi}$ is also a surjection whenever $\mathcal{A}$ is a $\sigma$-unital $C^{*}$-algebra; see, for instance, [5], Proposition 6.8. As noted in [5], the assertion fails, even in the commutative case, if one drops the requirement that $\mathcal{A}$ should be $\sigma$-unital. Observe that in the commutative case $\mathcal{A}=C_{0}(X), \mathcal{B}=C_{0}(Y)$ with $Y$ closed in $X$, we have $M(\mathcal{A})=C_{b}(X)$ and $M(\mathcal{B})=C_{b}(Y)$. Now the fact that any function in $M(\mathcal{B})_{\mathrm{sa}}=C_{b}(Y, \boldsymbol{R})$ lifts to a function in $M(\mathcal{A})_{\mathrm{sa}}=C_{b}(X, \boldsymbol{R})$ explains the name of the theorem.

The aim of this note is to generalize the noncommutative Tietze extension theorem to Hilbert $C^{*}$-modules. However, we note: although our Theorem 2 generalizes the noncommutative Tietze theorem, our proof uses that result. The proof of Theorem 2 also relies on our previous article [2] where the Hilbert $C^{*}$-module version of the multiplier algebra is constructed.

A (right) Hilbert $C^{*}$-module over a $C^{*}$-algebra $\mathcal{A}$ is a right $\mathcal{A}$-module $V$ equipped with an $\mathcal{A}$-valued inner product $\langle\cdot, \cdot\rangle$ such that $V$ is a Banach space with the norm $\|v\|=\|\langle v, v\rangle\|^{1 / 2}$. We assume that the linear structures on $\mathcal{A}$ and $V$ are compatible in the sense that $(\lambda v) a=\lambda(v a)=v(\lambda a)$ for all $v \in V, a \in \mathcal{A}, \lambda \in C$. $V$ is said to be a full Hilbert $\mathcal{A}$-module when the ideal $\langle V, V\rangle$ (= the closed linear span of all elements in $\mathcal{A}$ of the form $\langle x, y\rangle, x, y \in V)$ coincides with $\mathcal{A}$.

Received by the editors December 3, 2002 and, in revised form, July 11, 2003.

2000 Mathematics Subject Classification. Primary 46C50; Secondary 46L08.

Key words and phrases. $C^{*}$-algebra, Tietze extension theorem, Hilbert $C^{*}$-module.

(C)2004 American Mathematical Society 
If $V$ and $W$ are Hilbert $\mathcal{A}$-modules, we denote by $\boldsymbol{B}(V, W)$ the Banach space of all adjointable operators from $V$ to $W$. The ideal of "compact" operators (generated by all operators of the form $\left.\theta_{x, y}, \theta_{x, y}(v)=x\langle y, v\rangle\right)$ from $V$ to $W$ is denoted by $\boldsymbol{K}(V, W)$. When $V=W$ we write $\boldsymbol{B}(V)$ and $\boldsymbol{K}(V)$ instead of $\boldsymbol{B}(V, V)$ and $\boldsymbol{K}(V, V)$, respectively. If a $C^{*}$-algebra $\mathcal{A}$ is regarded as a Hilbert $\mathcal{A}$-module with the inner product $\langle a, b\rangle=a^{*} b$, then $\mathcal{A} \simeq \boldsymbol{K}(\mathcal{A})$ and $M(\mathcal{A}) \simeq \boldsymbol{B}(\mathcal{A})$. The corresponding identifications $a \leftrightarrow T_{a}$ and $m \leftrightarrow T_{m}$, with $T_{a}$ and $T_{m}$ denoting the left translations by $a \in \mathcal{A}$ resp. $m \in M(\mathcal{A})$, will be used freely.

For general facts about Hilbert $C^{*}$-modules the reader is referred to [5] and [7].

Given two Hilbert $C^{*}$-modules $V$ and $W$ over $\mathcal{A}$ and $\mathcal{B}$, respectively, we say that a map $\Phi: V \rightarrow W$ is a morphism of Hilbert $C^{*}$-modules if there exists a morphism of $C^{*}$-algebras $\varphi: \mathcal{A} \rightarrow \mathcal{B}$ such that $\langle\Phi(x), \Phi(y)\rangle=\varphi(\langle x, y\rangle)$ is satisfied for all $x$ and $y$ in $V$. When the underlying morphism $\varphi$ has to be emphasized, the map $\Phi$ will be called a $\varphi$-morphism. It is known that each $\varphi$-morphism is necessarily a linear contraction satisfying $\Phi(x a)=\Phi(x) \varphi(a), x \in V, a \in \mathcal{A}$, such that $\operatorname{Ker} \Phi=V_{\operatorname{Ker} \varphi}$, i.e. the kernel of $\Phi$ is the ideal submodule of $V$ associated to the kernel of $\varphi$. In particular, if $V$ is full, then $\Phi$ is an injection if and only if $\varphi$ is an injection.

As a typical example, consider an ideal $\mathcal{I}$ of $\mathcal{A}$ and take the corresponding ideal submodule $V_{\mathcal{I}}$ of a Hilbert $\mathcal{A}$-module $V$. Then the quotient map $q: V \rightarrow V / V_{\mathcal{I}}$ is a $\pi$-morphism of Hilbert $C^{*}$-modules (with $\pi: \mathcal{A} \rightarrow \mathcal{A} / \mathcal{I}$ denoting the quotient map of the underlying $C^{*}$-algebra).

A surjective $\varphi$-morphism $\Phi: V \rightarrow W$ is a unitary operator of Hilbert $C^{*}$-modules if the underlying morphism $\varphi$ is an injection. If this is the case we say that $V$ and $W$ are unitarily equivalent Hilbert $C^{*}$-modules. Unitary equivalence of full Hilbert $C^{*}$-modules is an equivalence relation.

For these and other facts concerning morphisms of Hilbert $C^{*}$-modules we refer the reader to [1].

In the present note we shall be concerned with extensions of morphisms of Hilbert $C^{*}$-modules. But first we need to describe the concept of the extended (multiplier) module.

Let $V$ be a full Hilbert $C^{*}$-module over a (non-unital) $C^{*}$-algebra $\mathcal{A}$. Denote by $V_{d}=\boldsymbol{B}(\mathcal{A}, V)$ the Hilbert $C^{*}$-module over the multiplier algebra $M(\mathcal{A})$ consisting of all adjointable maps from $\mathcal{A}$ to $V$ with the inner product $\langle r, s\rangle=r^{*} s$. Let $\Gamma: V \rightarrow V_{d}$ be defined by $\Gamma(v)=r_{v}$ where $r_{v}: \mathcal{A} \rightarrow V$ denotes the "multiplier" $r_{v}(a)=v a$. Then $\left(V_{d}, M(\mathcal{A}), \Gamma\right)$ is an extension of $V$ in the following sense: if we identify $v$ in $V$ with $\Gamma(v)$ in $V_{d}$, then $V$ is an ideal submodule of $V_{d}$ corresponding to the ideal $\mathcal{A}$ of $M(\mathcal{A})$. The extended module $V_{d}$ has the following universal property: Let $\varphi: \mathcal{A} \rightarrow \mathcal{B}$ be an injective morphism of $C^{*}$-algebras such that $\varphi(\mathcal{A})$ is an (essential) ideal in a $C^{*}$-algebra $\mathcal{B}$, and let $\lambda: \mathcal{B} \rightarrow M(\mathcal{A})$ be the resulting morphism such that $\lambda \varphi$ is the identity on $\mathcal{A}$. Let $W$ be a Hilbert $\mathcal{B}$-module. Suppose that $\Phi: V \rightarrow W$ is a $\varphi$-morphism of Hilbert $C^{*}$-modules with $\Phi(V)=V \varphi(\mathcal{A})$, so that $(W, \mathcal{B}, \Phi)$ is another (essential) extension of $V$. Then there exists a $\lambda$-morphism $\Lambda: W \rightarrow V_{d}$ such that $\Lambda \Phi=\Gamma$. Since the maps $\lambda$ and $\Lambda$ are injections precisely when $\varphi(\mathcal{A})$ is an essential ideal in $\mathcal{B}$, this shows that $V_{d}$ is the largest essential extension of $V$ ([2], Theorem 1.1).

Furthermore, given an essential extension $(W, \mathcal{B}, \Phi)$ of $V$, we define a (variant of) strict topology $\tau_{V}$ on $W$ by the family of seminorms $w \mapsto\|\langle\Phi(v), w\rangle\|, v \in V$, and $w \mapsto\|w \varphi(a)\|, a \in \mathcal{A}$. Note in passing that in the case $V=\mathcal{A}$ the extended 
module $V_{d}$ is nothing else than the multiplier algebra $M(\mathcal{A})$ and $\tau_{V}$ coincides with the usual $\left(C^{*}\right)$ strict topology on $M(\mathcal{A})$.

It is proved in [2] that $V$ is strictly dense in $V_{d}$ and that $V_{d}$ is complete with respect to the strict topology $\tau_{V}$. Moreover, each essential extension of $V$ complete with respect to $\tau_{V}$ is unitarily equivalent to $V_{d}$.

Finally, as observed in [2], $V_{d}$ coincides (up to the identification $v \leftrightarrow r_{v}$ ) with $V$ whenever either $\mathcal{A}$ or $\boldsymbol{K}(V)$ is a unital $C^{*}$-algebra.

All of this shows that $V_{d}$ can be regarded as the Hilbert $C^{*}$-module version of the multiplier algebra.

Suppose now that we are given a surjective $\varphi$-morphism $\Phi: V \rightarrow W$ of full Hilbert modules. Regarding $V_{d}$ and $W_{d}$ as their "multiplier" modules one may ask: is there any morphism of Hilbert $C^{*}$-modules $\bar{\Phi}: V_{d} \rightarrow W_{d}$ extending $\Phi$ in the sense $\bar{\Phi}\left(r_{v}\right)=r_{\Phi(v)}, \forall v \in V$ ?

Observe that this condition determines uniquely the underlying morphism of $C^{*}$ algebras $\psi: M(\mathcal{A}) \rightarrow M(\mathcal{B})$. Indeed, $\left\langle r_{v}, r_{v}\right\rangle=\langle v, v\rangle, \forall v \in V$; hence $\langle\bar{\Phi}(r), \bar{\Phi}(r)\rangle=$ $\psi(\langle r, r\rangle), \forall r \in V_{d}$ implies $\psi(\langle v, v\rangle)=\psi\left(\left\langle r_{v}, r_{v}\right\rangle\right)=\left\langle\bar{\Phi}\left(r_{v}\right), \bar{\Phi}\left(r_{v}\right)\right\rangle=\langle\Phi(v), \Phi(v)\rangle=$ $\varphi(\langle v, v\rangle), \forall v \in V$; thus $\psi$ must coincide with the canonical extension $\bar{\varphi}$ of $\varphi$.

Secondly, one would like to know when the extension $\bar{\Phi}$ of $\Phi$ is also a surjection.

Before stating main results of the paper we note that a related discussion can be found in [4. There multiplier modules of Hilbert $C^{*}$-bimodules are defined in a purely algebraic manner and a variant of the Tietze extension theorem for homomorphisms of Hilbert $C^{*}$-bimodules is obtained.

We end this introductory section by stating two main results of this note: the first one ensures the existence of the canonical extension $\bar{\Phi}$ of a surjective morphism $\Phi$, while the other serves as the Hilbert $C^{*}$-module version of the noncommutative Tietze extension theorem.

Proposition 1. Let $\Phi: V \rightarrow W$ be a surjective morphism of full Hilbert $C^{*}$ modules. Then there exists a unique morphism of Hilbert $C^{*}$-modules $\bar{\Phi}: V_{d} \rightarrow W_{d}$ extending $\Phi$ in the sense that $\bar{\Phi}\left(r_{v}\right)=r_{\Phi(v)}, \forall v \in V$. The map $\bar{\Phi}$ is the continuation of $\Phi$ with respect to the pair of strict topologies on $V_{d}$ and on $W_{d}$, hence uniquely determined.

Theorem 2. Let $V$ be a full countably generated Hilbert $C^{*}$-module over a $\sigma$ unital $C^{*}$-algebra, and let $W$ be another full Hilbert $C^{*}$-module. Then the canonical extension $\bar{\Phi}: V_{d} \rightarrow W_{d}$ of a surjective morphism $\Phi: V \rightarrow W$ is also a surjection.

Observe that the above theorem covers the case of $C^{*}$-algebras (simply by taking $V=\mathcal{A}, W=\mathcal{B}$ and $\Phi=\varphi$ ), so one cannot conclude that an extension $\bar{\Phi}$ is a surjection without some additional assumptions. On the other hand, by [5], Proposition 6.7 a full Hilbert $C^{*}$-module $V$ is countably generated if and only if $\boldsymbol{K}(V)$ is a $\sigma$-unital $C^{*}$-algebra. Hence in the basic case $V=\mathcal{A}$ the first hypothesis in Theorem 2, namely that $V$ should be countably generated, is ensured (via the fact $\boldsymbol{K}(\mathcal{A})=\mathcal{A}$ ) by the assumption that $\mathcal{A}$ is $\sigma$-unital.

\section{Proofs}

Since the proof of Proposition 1 effectively requires work in the quotient $V / \operatorname{Ker} \Phi$, we first recall from [1] some facts concerned with quotients of Hilbert $C^{*}$-modules. Let $V$ be a full $\mathcal{A}$-module, and let $\mathcal{I}$ be an ideal in $\mathcal{A}$. The associated ideal submodule $V_{\mathcal{I}}$ is defined as the closed linear span of the set $V \mathcal{I}=\{v a: v \in V, a \in \mathcal{I}\}$. 
After applying the Hewitt-Cohen factorization ([6], Proposition 2.31) one finds

$$
V_{\mathcal{I}}=V \mathcal{I}=\{v \in V:\langle v, x\rangle \in \mathcal{I}, \forall x \in V\} .
$$

Now consider the quotient Hilbert $\mathcal{A} / \mathcal{I}$-module $V / V_{\mathcal{I}}$ with $q: V \rightarrow V / V_{\mathcal{I}}$ denoting the quotient map, and take an arbitrary adjointable operator $T \in \boldsymbol{B}(V)$. Since $V_{\mathcal{I}}$ is obviously invariant for $T$ (by (1)), there is a well-defined operator $\hat{T}$ on $V / V_{\mathcal{I}}$ given by $\hat{T}(q(v))=q(T v)$. Note also $\hat{\theta}_{x, y}^{\wedge}=\theta_{q(x), q(y)}, \forall x, y \in V$. It is proved in Corollary 1.18 from [1] that the map $\beta: \boldsymbol{B}(V) \rightarrow \boldsymbol{B}\left(V / V_{\mathcal{I}}\right), \beta(T)=\hat{T}$ is a morphism of $C^{*}$-algebras such that $\beta(\boldsymbol{K}(V))=\boldsymbol{K}\left(V / V_{\mathcal{I}}\right)$. In particular, if $V$ is countably generated, then $\boldsymbol{K}(V)$ is a $\sigma$-unital $C^{*}$-algebra ([5], Proposition 6.7), and by the noncommutative Tietze extension theorem, $\beta$ is a surjection.

In the basic case $V=\mathcal{A}$ the corresponding map will be denoted by $\beta_{0}$. Observe that $\beta_{0}\left(T_{a}\right)=T_{\pi(a)}$ for $a \in \mathcal{A}$, so that $\beta_{0}$ restricted to "compact" operators coincides with the quotient map $\pi$ (up to the identification $a \leftrightarrow T_{a}$ ). Consequently, applying $\beta_{0}$ to multipliers $T_{m}$ one gets the canonical extension of the quotient map $\pi$.

Proposition 3. Let $V$ be a full $\mathcal{A}$-module, and let $\mathcal{I}$ be an ideal in $\mathcal{A}$. The map $\tau: V_{d} \rightarrow\left(V / V_{\mathcal{I}}\right)_{d}$ defined by $\tau(r)=\hat{r}, \hat{r}(\pi(a))=q(r(a))$ is a $\beta_{0}$-morphism of Hilbert $C^{*}$-modules satisfying $\tau\left(r_{x}\right)=r_{q(x)}, \forall x \in V$. The map $\tau$ is continuous with respect to the pair of strict topologies on $V_{d}$ and on $\left(V / V_{\mathcal{I}}\right)_{d}$.

Proof. First, $\hat{r}$ is well defined: $\pi(a)=\pi(b) \Rightarrow a-b \in \mathcal{I} \Rightarrow\langle r(a-b), x\rangle=$ $\left\langle a-b, r^{*}(x)\right\rangle=(a-b)^{*} r^{*}(x) \in \mathcal{I}, \forall x \in V$. Using (1) we now conclude that $r(a-b) \in V_{\mathcal{I}}$, hence $q(r(a))=q(r(b))$.

Secondly, $\tau$ is well defined, i.e. $\hat{r}$ is an adjointable map. Indeed, for an adjointable map $l: V \rightarrow \mathcal{A}$ we define $\hat{l}: V / V_{\mathcal{I}} \rightarrow \mathcal{A} / \mathcal{I}$ by $\hat{l}(q(v))=\pi(l(v))$. By a straightforward calculation (which we omit) one verifies that $\hat{l}$ is also an adjointable map and $(\hat{r})^{*}=\left(r^{*}\right)^{\wedge}$.

We next show that $\tau$ is a $\beta_{0}$-morphism of Hilbert $C^{*}$-modules; by definition we have to check that $\langle\tau(r), \tau(s)\rangle=\beta_{0}(\langle r, s\rangle)$. Again, this is a routine verification: $\langle\tau(r), \tau(s)\rangle(\pi(a))=\tau(r)^{*} \tau(s)(\pi(a))=\tau(r)^{*}(q(s(a)))=\pi\left(r^{*}(s(a))\right)=\pi\left(\left(r^{*} s\right)(a)\right)$ $=\pi(\langle r, s\rangle(a))=\beta_{0}(\langle r, s\rangle)(\pi(a))$.

The equality $\tau\left(r_{x}\right)=r_{q(x)}, x \in V$ is evident, and the proof will be finished by showing that $\tau$ is strictly continuous. Assume $r=\left(\right.$ st.) $\lim _{j} r_{j}$ in $V_{d}$. This means

$$
\left\langle r, r_{x}\right\rangle=\lim _{j}\left\langle r_{j}, r_{x}\right\rangle, \forall x \in V
$$

and

$$
r T_{a}=\lim _{j} r_{j} T_{a}, \forall a \in \mathcal{A} .
$$

We must obtain analogous relations in order to prove $\tau(r)=$ (st.) $\lim _{j} \tau\left(r_{j}\right)$. This is immediate from the properties of the map $\tau: \lim _{j}\left\langle\tau\left(r_{j}\right), r_{q(x)}\right\rangle=\lim _{j}\left\langle\tau\left(r_{j}\right), \tau\left(r_{x}\right)\right\rangle$ $=\lim _{j} \beta_{0}\left(\left\langle r_{j}, r_{x}\right\rangle\right)=$ (by (2) ) $\beta_{0}\left(\left\langle r, r_{x}\right\rangle\right)=\left\langle\tau(r), \tau\left(r_{x}\right)\right\rangle=\left\langle\tau(r), r_{q(x)}\right\rangle, \forall x \in V$. For the proof of the remaining part observe that $\beta_{0}$ is surjective on "compact" operators and $\tau$ is $\beta_{0}$-linear and norm-continuous: $\lim _{j} \tau\left(r_{j}\right) \beta_{0}\left(T_{a}\right)=\lim _{j} \tau\left(r_{j} T_{a}\right)=$ (by (3)) $\tau\left(r T_{a}\right)=\tau(r) \beta_{0}\left(T_{a}\right), \forall \beta_{0}\left(T_{a}\right) \in \boldsymbol{K}(\mathcal{A} / \mathcal{I})$. 
Remark 4. Note that the last part of the preceding proof shows that any morphism $\tau^{\prime}: V_{d} \rightarrow\left(V / V_{\mathcal{I}}\right)_{d}$ satisfying $\tau^{\prime}\left(r_{x}\right)=r_{q(x)}, \forall x \in V$ is strictly continuous. Since $V_{d}$ and $\left(V / V_{\mathcal{I}}\right)_{d}$ are the strict completions of the sets $\left\{r_{x}: x \in V\right\}$, resp. $\left\{r_{q}(x)\right.$ : $x \in V\}$ (2], Theorem 1.5), we conclude that $\tau$ is uniquely determined with the property $\tau\left(r_{x}\right)=r_{q(x)}, \forall x \in V$.

Proof of Proposition 1. Suppose that $V$ and $W$ are full Hilbert $C^{*}$-modules over $\mathcal{A}$ and $\mathcal{B}$, respectively, and that $\Phi: V \rightarrow W$ is a surjective morphism. Note that, since $V$ and $W$ are full, the underlying morphism of $C^{*}$-algebras $\varphi: \mathcal{A} \rightarrow \mathcal{B}$ is also a surjection. Passing through the corresponding quotients we conclude: $V_{\operatorname{Ker} \varphi}$ and $W$ are unitarily equivalent Hilbert $C^{*}$-modules; the map $q(v) \mapsto \Phi(v)$ induced by $\Phi$ serves as a unitary operator (with the underlying isomorphism of $C^{*}$-algebras given by $\pi(a) \mapsto \varphi(a))$.

It is obvious that for unitarily equivalent Hilbert $C^{*}$-modules $W$ and $W^{\prime}$ the extended modules $W_{d}$ and $W_{d}^{\prime}$ are also unitarily equivalent. Combining this with the preceding proposition we obtain Proposition 10 Observe additionally that $\bar{\Phi}$ is given by the formula $\bar{\Phi}(r)(\varphi(a))=\Phi(r(a)), r \in V_{d}$.

Remark 5. Since unitarily equivalent Hilbert $C^{*}$-modules have isomorphic $C^{*}$ algebras of "compact" and adjointable operators, we may also apply the discussion before Proposition 3 to conclude: there is a morphism of $C^{*}$-algebras $\Phi^{+}: \boldsymbol{B}(V) \rightarrow$ $\boldsymbol{B}(W)$ such that $\Phi^{+}\left(\theta_{x, y}\right)=\theta_{\Phi(x), \Phi(y)}, \forall x, y \in V$ and $\Phi^{+}(\boldsymbol{K}(V))=\boldsymbol{K}(W)$. The map $\Phi^{+}$is a surjection when $V$ is countably generated.

Note that $\Phi$ is supported by $\Phi^{+}$in the same way as by the underlying map $\varphi$. Namely, if we consider the left Hilbert $C^{*}$-module structure on $V$ over $\boldsymbol{K}(V)$ with the inner product $[x, y]=\theta_{x, y}$ then, by the above description of the action of $\Phi^{+}$on elementary operators, we have $[\Phi(x), \Phi(y)]=\Phi^{+}([x, y]), \forall x, y \in V$. As an immediate consequence we also note that $\Phi(T x)=\Phi^{+}(T) \Phi(x), \forall T \in \boldsymbol{K}(V), \forall x \in$ $V$.

We now turn to the proof of Theorem 2 Our approach consists of passing to the linking algebras and making use of the noncommutative Tietze theorem for $C^{*}$-algebras.

Let $V$ be a full Hilbert $\mathcal{A}$-module. Recall from 3] (see also Lemma 2.32 and Corollary 3.21 in [6]) that the linking algebra $L(V)$ of $V$ can be described in terms of a Hilbert $\mathcal{A}$-module $\mathcal{A} \oplus V$ :

$$
L(V)=\boldsymbol{K}(\mathcal{A} \oplus V)=\left[\begin{array}{cc}
\boldsymbol{K}(\mathcal{A}) & \boldsymbol{K}(V, \mathcal{A}) \\
\boldsymbol{K}(\mathcal{A}, V) & \boldsymbol{K}(V)
\end{array}\right] .
$$

Since each operator in $\boldsymbol{K}(\mathcal{A}, V)$ is of the form $r_{x}$ for some $x \in V$, we have

$$
L(V)=\left\{\left[\begin{array}{cc}
T_{a} & r_{y}^{*} \\
r_{x} & T
\end{array}\right]: a \in \mathcal{A}, x, y \in V, T \in \boldsymbol{K}(V)\right\} .
$$

Suppose that $\Phi: V \rightarrow W$ is a surjective $\varphi$-morphism of Hilbert $C^{*}$-modules. Then there is a map $\rho=\rho_{\Phi}$ of the corresponding linking algebras:

$$
\rho_{\Phi}: L(V) \rightarrow L(W), \rho_{\Phi}\left(\left[\begin{array}{cc}
T_{a} & r_{y}^{*} \\
r_{x} & T
\end{array}\right]\right)=\left[\begin{array}{cc}
T_{\varphi(a)} & r_{\Phi(y)}^{*} \\
r_{\Phi(x)} & \Phi^{+}(T)
\end{array}\right] .
$$

It is proved in Theorem 2.15 in [1] that $\rho_{\Phi}$ is a morphism of $C^{*}$-algebras. In fact, it turns out that $\rho_{\Phi}$ is the restriction of the map $(\varphi \oplus \Phi)^{+}$to "compact" operators, 
where $\varphi \oplus \Phi: \mathcal{A} \oplus V \rightarrow \mathcal{B} \oplus W$ is a $\varphi$-morphism of Hilbert $C^{*}$-modules obtained by applying $\varphi$ and $\Phi$ componentwise.

Proof of Theorem Q Let $\Phi: V \rightarrow W$ be a surjective $\varphi$-morphism of full Hilbert $C^{*}$-modules with $\varphi: \mathcal{A} \rightarrow \mathcal{B}$, and let $V$ be countably generated and $\mathcal{A} \sigma$-unital. Since $V$ and $W$ are full, $\varphi$ is also a surjection. The map $\Phi^{+}$is surjective by Remark 5 and now the preceding paragraph shows that $\rho_{\Phi}: \boldsymbol{K}(\mathcal{A} \oplus V) \rightarrow \boldsymbol{K}(\mathcal{B} \oplus W)$ is also a surjective map. Denote by

$$
\overline{\rho_{\Phi}}: \boldsymbol{B}(\mathcal{A} \oplus V) \rightarrow \boldsymbol{B}(\mathcal{B} \oplus W)
$$

the canonical extension of $\rho_{\Phi}$. Since $V$ is by assumption a countably generated Hilbert $\mathcal{A}$-module and $\mathcal{A}$ is a $\sigma$-unital $C^{*}$-algebra, $\mathcal{A} \oplus V$ is also countably generated. By [5], Proposition $6.7, \boldsymbol{K}(\mathcal{A} \oplus V)$ is then a $\sigma$-unital $C^{*}$-algebra. Thus, by the noncummatative Tietze extension theorem, the map $\overline{\rho_{\Phi}}$ is also a surjection. Now observe that (7) can be rewritten as

$$
\overline{\rho_{\Phi}}:\left[\begin{array}{cc}
\boldsymbol{B}(\mathcal{A}) & \boldsymbol{B}(V, \mathcal{A}) \\
\boldsymbol{B}(\mathcal{A}, V) & \boldsymbol{B}(V)
\end{array}\right] \rightarrow\left[\begin{array}{cc}
\boldsymbol{B}(\mathcal{B}) & \boldsymbol{B}(W, \mathcal{B}) \\
\boldsymbol{B}(\mathcal{B}, W) & \boldsymbol{B}(W)
\end{array}\right],
$$

or, recognizing $\boldsymbol{B}(\mathcal{A}, V)=V_{d}$ and $\boldsymbol{B}(\mathcal{B}, W)=W_{d}$,

$$
\overline{\rho_{\Phi}}:\left[\begin{array}{cc}
\boldsymbol{B}(\mathcal{A}) & \left(V_{d}\right)^{*} \\
V_{d} & \boldsymbol{B}(V)
\end{array}\right] \rightarrow\left[\begin{array}{cc}
\boldsymbol{B}(\mathcal{B}) & \left(W_{d}\right)^{*} \\
W_{d} & \boldsymbol{B}(W)
\end{array}\right] .
$$

The remaining part of the proof consists of showing that the extended morphism $\overline{\rho_{\Phi}}$ preserves matricial corners; this will imply that $\overline{\rho_{\Phi}}$ is in fact equal to the map $\rho_{\bar{\Phi}}$. The argument below is similar to the proof of Theorem 2.15 in [1] and uses strict continuity of $\overline{\rho_{\Phi}}$ and the fact that the original map $\rho_{\Phi}$ preserves corners.

Take an arbitrary multiplier $m \in M(\mathcal{A})$ interpreted as the left translation $T_{m} \in$ $\boldsymbol{B}(\mathcal{A})$. Let $\left(a_{n}\right)$ be an approximate unit for $\mathcal{A}$. We first claim that

$$
\left[\begin{array}{cc}
T_{m} & 0 \\
0 & 0
\end{array}\right]=\text { (st.) } \lim _{n}\left[\begin{array}{cc}
T_{m a_{n}} & 0 \\
0 & 0
\end{array}\right] \text {. }
$$

Indeed, taking arbitrary $\left[\begin{array}{cc}T_{a} & r_{y}^{*} \\ r_{x} & T\end{array}\right] \in \boldsymbol{K}(\mathcal{A} \oplus V)$, we must show that

$$
\begin{gathered}
{\left[\begin{array}{cc}
T_{a} & r_{y}^{*} \\
r_{x} & T
\end{array}\right]\left[\begin{array}{cc}
T_{m a_{n}} & 0 \\
0 & 0
\end{array}\right]=\left[\begin{array}{cc}
T_{a m a_{n}} & 0 \\
r_{x} T_{m a_{n}} & 0
\end{array}\right] \rightarrow\left[\begin{array}{cc}
T_{a m} & 0 \\
r_{x} T_{m} & 0
\end{array}\right]} \\
=\left[\begin{array}{cc}
T_{a} & r_{y}^{*} \\
r_{x} & T
\end{array}\right]\left[\begin{array}{cc}
T_{m} & 0 \\
0 & 0
\end{array}\right]
\end{gathered}
$$

and

$$
\begin{gathered}
{\left[\begin{array}{cc}
T_{m a_{n}} & 0 \\
0 & 0
\end{array}\right]\left[\begin{array}{cc}
T_{a} & r_{y}^{*} \\
r_{x} & T
\end{array}\right]=\left[\begin{array}{cc}
T_{m a_{n} a} & T_{m a_{n}} r_{y}^{*} \\
0 & 0
\end{array}\right] \rightarrow\left[\begin{array}{cc}
T_{m a} & T_{m} r_{y}^{*} \\
0 & 0
\end{array}\right]} \\
=\left[\begin{array}{cc}
T_{m} & 0 \\
0 & 0
\end{array}\right]\left[\begin{array}{cc}
T_{a} & r_{y}^{*} \\
r_{x} & T
\end{array}\right] .
\end{gathered}
$$

To verify the above two limit relations we need only to check that $r_{x} T_{m a_{n}} \rightarrow$ $r_{x} T_{m}$ and $T_{m a_{n}} r_{y}^{*} \rightarrow T_{m} r_{y}^{*}$. But, this follows easily from the fact that $\left(T_{a_{n}}\right)$ is an 
approximate unit:

$$
\begin{aligned}
\left\|r_{x} T_{m a_{n}}-r_{x} T_{m}\right\|^{2} & =\left\|r_{x}\left(T_{m} T_{a_{n}}-T_{m}\right)\right\|^{2}=\left\|\left(T_{m} T_{a_{n}}-T_{m}\right)^{*} r_{x}^{*} r_{x}\left(T_{m} T_{a_{n}}-T_{m}\right)\right\| \\
& =\left\|\left(T_{m} T_{a_{n}}-T_{m}\right)^{*} T_{\langle x, x\rangle}\left(T_{m} T_{a_{n}}-T_{m}\right)\right\| \\
& =\left\|T_{\langle x, x\rangle}^{1 / 2}\left(T_{m} T_{a_{n}}-T_{m}\right)\right\|^{2} \rightarrow 0 .
\end{aligned}
$$

Similarly, $\left\|T_{m} T_{a_{n}} r_{y}^{*}-T_{m} r_{y}^{*}\right\|^{2}=\left\|\left(T_{m} T_{a_{n}}-T_{m}\right) r_{y}^{*} r_{y}\left(T_{m} T_{a_{n}}-T_{m}\right)^{*}\right\| \rightarrow 0$.

Analogously, for arbitrary $S \in \boldsymbol{B}(V)$ and an approximate unit $\left(T_{n}\right)$ for $\boldsymbol{K}(V)$, we claim

$$
\left[\begin{array}{ll}
0 & 0 \\
0 & S
\end{array}\right]=\text { (st.) } \lim _{n}\left[\begin{array}{cc}
0 & 0 \\
0 & S T_{n}
\end{array}\right] .
$$

To obtain the last equality one has only to repeat the above verification (with the final limit relations concerned with the approximate unit in $\boldsymbol{K}(V)$ and the operators $\theta_{x, x}$ and $\left.\theta_{y, y}\right)$. We omit the details.

Now we apply $\overline{\rho_{\Phi}}$ to (10) and (11). Since $\overline{\rho_{\Phi}}$ is strictly continuous and extends $\rho_{\Phi}$, and since the canonical extensions $\bar{\varphi}$ and $\overline{\Phi^{+}}$are strictly continuous, we obtain from (10),

$$
\overline{\rho_{\Phi}}\left(\left[\begin{array}{cc}
T_{m} & 0 \\
0 & 0
\end{array}\right]\right)=\text { (st.) } \lim _{n} \rho_{\Phi}\left(\left[\begin{array}{cc}
T_{m a_{n}} & 0 \\
0 & 0
\end{array}\right]\right)=(\text { st. }) \lim _{n}\left[\begin{array}{cc}
T_{\varphi\left(m a_{n}\right)} & 0 \\
0 & 0
\end{array}\right] ;
$$

hence

$$
\overline{\rho_{\Phi}}\left(\left[\begin{array}{cc}
T_{m} & 0 \\
0 & 0
\end{array}\right]\right)=\left[\begin{array}{cc}
T_{\bar{\varphi}(m)} & 0 \\
0 & 0
\end{array}\right], \forall T_{m} \in \boldsymbol{B}(\mathcal{A}) .
$$

In the same way, starting from (II), we get

$$
\overline{\rho_{\Phi}}\left(\left[\begin{array}{ll}
0 & 0 \\
0 & S
\end{array}\right]\right)=\left[\begin{array}{cc}
0 & 0 \\
0 & \overline{\Phi^{+}}(S)
\end{array}\right], \forall S \in \boldsymbol{B}(V) .
$$

Now take arbitrary $r \in \boldsymbol{B}(\mathcal{A}, V)=V_{d}$ and put

$$
\overline{\rho_{\Phi}}\left(\left[\begin{array}{ll}
0 & 0 \\
r & 0
\end{array}\right]\right)=\left[\begin{array}{ll}
\rho_{11}(r) & \rho_{12}(r) \\
\rho_{21}(r) & \rho_{22}(r)
\end{array}\right] .
$$

Then

$$
\begin{aligned}
& \overline{\rho_{\Phi}}\left(\left[\begin{array}{ll}
0 & 0 \\
r & 0
\end{array}\right]^{*}\right) \overline{\rho_{\Phi}}\left(\left[\begin{array}{ll}
0 & 0 \\
r & 0
\end{array}\right]\right)=\left[\begin{array}{ll}
\rho_{11}(r)^{*} & \rho_{21}(r)^{*} \\
\rho_{12}(r)^{*} & \rho_{22}(r)^{*}
\end{array}\right]\left[\begin{array}{ll}
\rho_{11}(r) & \rho_{12}(r) \\
\rho_{21}(r) & \rho_{22}(r)
\end{array}\right] \\
& \times\left[\begin{array}{cc}
\rho_{11}(r)^{*} \rho_{11}(r)+\rho_{21}(r)^{*} \rho_{21}(r) & \rho_{11}(r)^{*} \rho_{12}(r)+\rho_{21}(r)^{*} \rho_{22}(r) \\
\rho_{12}(r)^{*} \rho_{11}(r)+\rho_{22}(r)^{*} \rho_{21}(r) & \rho_{12}(r)^{*} \rho_{12}(r)+\rho_{22}(r)^{*} \rho_{22}(r)
\end{array}\right],
\end{aligned}
$$

and also

$$
\overline{\rho_{\Phi}}\left(\left[\begin{array}{ll}
0 & 0 \\
r & 0
\end{array}\right]^{*}\right) \overline{\rho_{\Phi}}\left(\left[\begin{array}{ll}
0 & 0 \\
r & 0
\end{array}\right]\right)=\overline{\rho_{\Phi}}\left(\left[\begin{array}{cc}
r^{*} r & 0 \\
0 & 0
\end{array}\right]\right)=\left[\begin{array}{cc}
\bar{\varphi}\left(r^{*} r\right) & 0 \\
0 & 0
\end{array}\right] .
$$

Comparing (15) and (16) we conclude that $\rho_{12}(r)=0$ and $\rho_{22}(r)=0$. Similarly, by calculating $\overline{\rho_{\Phi}}\left(\left[\begin{array}{ll}0 & 0 \\ r & 0\end{array}\right]\right) \overline{\rho_{\Phi}}\left(\left[\begin{array}{ll}0 & 0 \\ r & 0\end{array}\right]^{*}\right)$ one finds $\rho_{11}(r)=0$ and, after all,

$$
\overline{\rho_{\Phi}}\left(\left[\begin{array}{ll}
0 & 0 \\
r & 0
\end{array}\right]\right)=\left[\begin{array}{cc}
0 & 0 \\
\Psi(r) & 0
\end{array}\right]
$$


where $\Psi: V_{d} \rightarrow W_{d}$ is a well-defined map of Hilbert $C^{*}$-modules. Since $\overline{\rho_{\Phi}}$ extends $\rho_{\Phi}$, the map $\Psi$ is an extension of $\Phi$. Additionally, (16) shows that $\Psi$ is a $\bar{\varphi}$ morphism of Hilbert $C^{*}$-modules. Since by Proposition $1 \bar{\Phi}: V_{d} \rightarrow W_{d}$ is the only morphism with these properties, we finally have $\Psi=\bar{\Phi}$.

This together with (12) and (13) gives a general formula for $\overline{\rho_{\Phi}}$ :

$$
\overline{\rho_{\Phi}}\left(\left[\begin{array}{cc}
T_{m} & s^{*} \\
r & S
\end{array}\right]\right)=\left[\begin{array}{cc}
T_{\bar{\varphi}}(m) & (\bar{\Phi}(s))^{*} \\
\bar{\Phi}(r) & \overline{\Phi^{+}}(S)
\end{array}\right],
$$

from which the surjectivity of $\bar{\Phi}$ is evident.

We end the paper with a result concerning strictly complete Hilbert $C^{*}$-modules. By definition, a Hilbert $\mathcal{A}$-module $V$ is said to be strictly complete if $V_{d}=V$, i.e. if each adjointable map $r: \mathcal{A} \rightarrow V$ is of the form $r=r_{v}, r_{v}(a)=v a$, for some $v$ in $V$. For the properties of strictly complete modules the reader is referred to [2].

Corollary 6. Let $V$ be a full strictly complete countably generated Hilbert $C^{*}$ module over a $\sigma$-unital $C^{*}$-algebra. Suppose that $\Phi: V \rightarrow W$ is a surjective morphism of full Hilbert $C^{*}$-modules. Then $W$ is also strictly complete. In particular, the quotient $V / V_{\mathcal{I}}$ over each ideal submodule $V_{\mathcal{I}}$ of $V$ is a strictly complete Hilbert $C^{*}$-module.

Proof. By the above theorem, $\bar{\Phi}: V_{d} \rightarrow W_{d}$ is a surjection. Thus each $r \in W_{d}$ has a preimage in $V_{d}$. Since $V_{d}=V$, there exists $x \in V$ such that $\bar{\Phi}\left(r_{x}\right)=r$; in other words, $r_{\Phi(x)}=r$.

\section{ACKNOWLEDGEMENT}

We thank the referee for a careful reading of the manuscript and for drawing our attention to the paper of Xiaochun Fang ([4]).

\section{REFERENCES}

[1] D. Bakić, B. Guljaš, On a class of module maps of Hilbert $C^{*}$-modules, Mathematical Communications 7(2002) No.2, 177-192. MR 1952758 (2003k:46084)

[2] D. Bakić, B. Guljaš, Extensions of Hilbert $C^{*}$-modules, to appear in Houston J. Math.

[3] L. G. Brown, P. Green, M. A. Rieffel, Stable isomorphism and strong Morita equivalence of $C^{*}$-algebras, Pacific J. Math, 71(1977), 349-363. MR 0463928 (57:3866)

[4] X. Fang, The realization of multiplier Hilbert bimodule on bidual space and Tietze extension theorem, Chinese Ann. Math Ser. B 21(2000), 375-380. MR 1788294 (2001h:46108)

[5] C. Lance, Hilbert $C^{*}$-modules: a toolkit for operator algebraists, London Math. Soc. Lecture Notes Series, vol. 210, Cambridge University Press, Cambridge, 1995. MR 1325694 (96k:46100)

[6] I. Raeburn, D. P. Williams, Morita equivalence and continuous-trace $C^{*}$-algebras, Mathematical Surveys and Monographs 60, AMS, 1998. MR 1634408 (2000c:46108)

[7] N. E. Wegge-Olsen, K-theory and $C^{*}$-algebras, Oxford University Press, Oxford, 1993. MR 1222415 (95c:46116)

Department of Mathematics, University of Zagreb, BijeničKa cesta 30, P.O.Box 335, 10002 ZaGreb, Croatia

E-mail address: bakic@math.hr 